\title{
Design of A Side-feeding Resistance-loaded Antipodal Vivaldi Antenna
}

\author{
Haibo Tang \\ School of Information and Electronic Engineering \\ Beijing Institute of Technology \\ Beijing,China \\ seafish789@163.com
}

\author{
Xiaozhong Shui \\ School of Information and Electronic Engineering \\ Beijing Institute of Technology \\ Beijing,China \\ shuixiaozhong@163.com
}

\begin{abstract}
This paper presented the design of a side-feeding resistance-loaded antipodal Vivaldi antenna. The dimensions of the antenna have been simulated and analyzed with highfrequency electromagnetic simulation software HFSS. A sidefeeding antipodal Vivaldi antenna for $0.38 \sim 4.8 \mathrm{GHz}$ was designed and fabricated. And its impedance and radiation characteristics have been measured. The results show that the Vivaldi antenna can match well within the $0.38-4.8 \mathrm{GHz}$ and has a good radiating directivity. Compared with traditional Vivaldi antenna, a side-feeding and resistance-loaded antipodal antenna can reduce the antenna's size effectively on the one hand and an increase of the parasitic patch can improve antenna's gain on the other hand.
\end{abstract}

Keywords-antipodal Vivaldi antenna;side-feeding;resistanceloaded

\section{INTRODUCTION}

Ultra-wideband antenna is widely used in the field of electronic communicatios, especially in mobile communications that have been rapidly developed. Bandwidth is a finite resource and how to use the finite bandwidth more efficiently is an urgent problem to be solved in modern communication systems.

Relative bandwidth is narrow in traditional communication systems and radar and can no longer meet the growing demands. The Vivaldi antenna is a slot antenna proposed by Gibson in 1979[1], which includes a slotline that is narrow in the end of antenna and open widely by a period of exponential slot-line in the other end of the antenna.This antenna has a simple structure and ultrawideband characteristic.In addition, the Vivaldi antenna has some other advantages such as low lobe level,high gain and adjustable beam width[2-4].

Antipodal Vivaldi antenna has a different structure compared with traditional that-the two metal claddings forming a slot line in traditional Vivaldi antenna are printed on the upper and lower dielectric layer surface respecttively[5].Antipodal Vivaldi has lower input impedance than traditional Vivaldi antenna and is easy to match feed line with characteristic impedance of $50 \mathrm{ohm}$.In addition, its mechanical properties are more stable and is more suitable for engineering maintenance.

This paper designed a $0.38 \mathrm{GHz}-4.8 \mathrm{GHz}$ antipodal Vivaldi antenna,the antenna has the advantage of miniaturization through side-feeding and resistance-loaded measures.

\section{ANTENNA DESIGN}

The basic geometry of the designed antipodal Vivaldi antenna in this paper is shown in Fig.1.The same with traditional Vivaldi antenna,this antenna can be divided into three sections:feed section, transition and radiating section. The feed section is a connection with the microstrip line on the top of substrate and the metal cladding on the same side contacted directly, the transition section is a parallel slot-line which connects the feed section and the radiating section. The radiating section is consists of two tapered lines that is determind by the exponential function shown in (1).

$$
y=c_{1} e^{R_{x}}+c_{2}
$$

where

$$
c_{1}=\frac{y_{2}-y_{1}}{e^{R x_{2}}-e^{R x_{1}}},
$$

and

$$
C_{2}=\frac{y_{1} e^{R x_{2}}-y_{2} e^{R x_{1}}}{e^{R x_{2}}-e^{R x_{1}}},
$$

$\mathrm{R}$ stands for the exponent of exponential function.

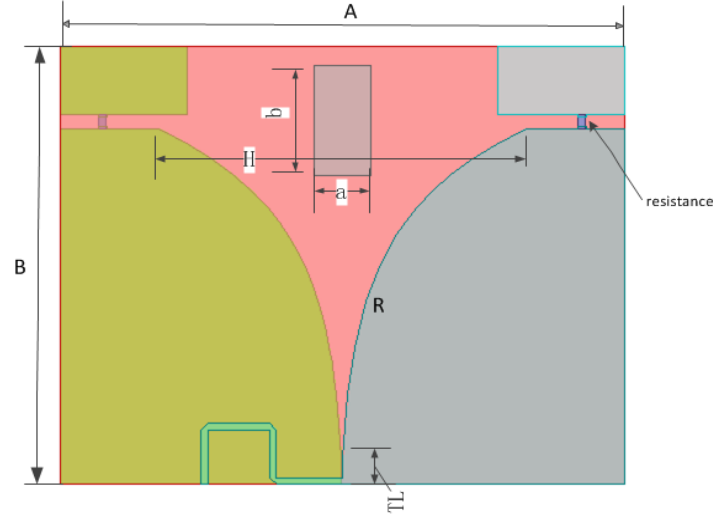

Figure 1. Geometry of the designed Vivaldi antenna

Parallel microstrip line feeding structure occupies part of the antenna area in the traditional Vivaldi antenna.In order to make use of the substrate and reduce the antenna size, a sidefeeding structure is designed in this paper.It means that feeding microstrip line contacts the metal cladding from the side direction ,not from the front and the metal cladding on the other side of substrate is used as the ground plane of the microstrip line. 


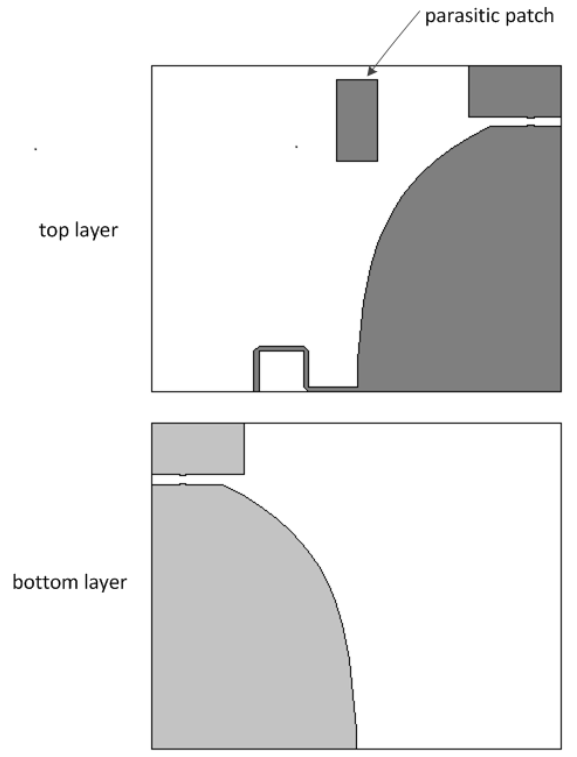

Figure 2. Structure of different layers in the designed antenna

Fig.2 shows the structure of different layers in the designed antenna.According to the Vivaldi antenna theory[6],in order to make the low-frequency band to obtain a good match,the antenna's Aperture Width must be greater than half the wavelength of the minimum frequency in the band.Therefore,in order to make the antenna miniaturization, it is necessary to take special measures in reducing the antenna's Aperture Width without affecting the match in the low-frequency band at the same time.A resistance-loaded method is adopted in this paper,that is adding two metal face at the end of the slotline and loading a resistance between the two gaps respectively.There is a parasitic patch on the substrate, which not only improves the gain and but also solves the flaw of radiation pattern in highfrequency band.

The design parameters of the antenna include the width of the antenna(A),Aperture Width(H),length of the antenna(B),length of the transition section(TL), value of resistance(RR), the width of the parasitic patch(a), the length of the parasitic patch(b), exponent of the tapered line(R).The designed antenna has been simulated by using the High Frequency Structure Simulator(HFSS) based on finite element method.The substrate is chosen to be FR4, which has the thickness of $2 \mathrm{~mm}$, a dielectric constant of 4.4 .

\section{RESULTS}

The antenna is optimized to have the dimension of $200 \mathrm{~mm} \times 160 \mathrm{~mm}$. And the parameters are set as follows: $\mathrm{H}=150 \mathrm{~mm}, \mathrm{TL}=10 \mathrm{~mm}, \mathrm{a}=20 \mathrm{~mm}, \mathrm{~b}=40 \mathrm{~mm}, \mathrm{R}=0.03, \mathrm{RR}=100 \Omega$. Because the wavelength corresponding to $0.38 \mathrm{GHz}$ is about $780 \mathrm{~mm}$, the Aperture Width $\mathrm{H}$ should be greater than $390 \mathrm{~mm}$ according to the Vivaldi theory as described above.However,actually the designed Aperture Width $\mathrm{H}$ is only $150 \mathrm{~mm}$ in this paper.So the antenna size is reduced successfully.Fig. 3 shows the fabricated antipodal Vivaldi antenna.

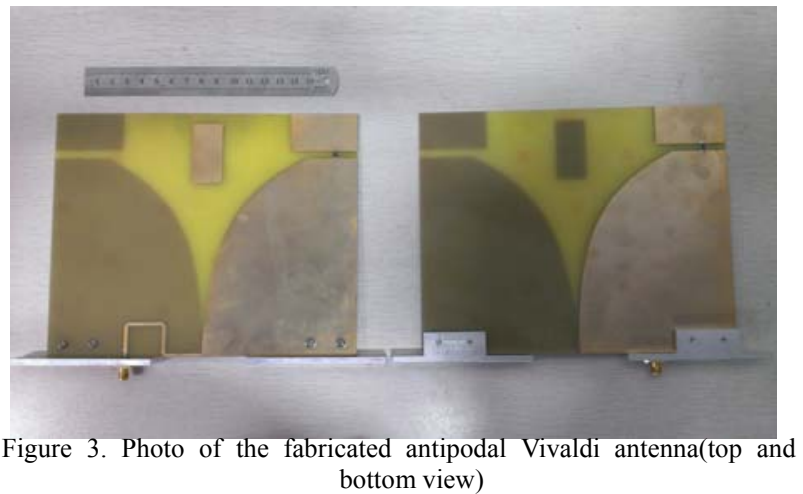

The simulated and measured results of the return loss are compared in the Fig.4.It can be seen that the return loss is less than $-10 \mathrm{~dB}$ from $0.38 \mathrm{GHz}$ to $4.8 \mathrm{GHz}$ in the simulated result,demonstrating its wideband performance.And the measured result is accordance with the simulated result,except at some low frequencies.

The curve of simulated gain varing with frequency can be seen from figure 5, which shows that the basic trend of gain is increasing along with frequency in the beginning and then decreasing in the end. The realized gain of the antenna has been tested at some frequencies listed in the Table 1.The gain declines in the measurement compared to the simulated results and the reason may be the dielectric loss and feeder loss introduced by the welding of the SMA connector and some other mechanical loss.

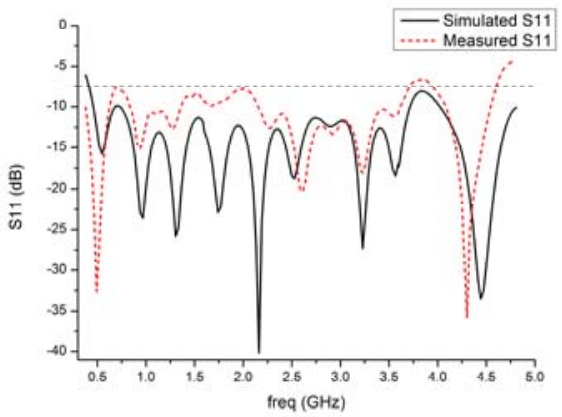

Figure 4. Simulated and measured results of return loss

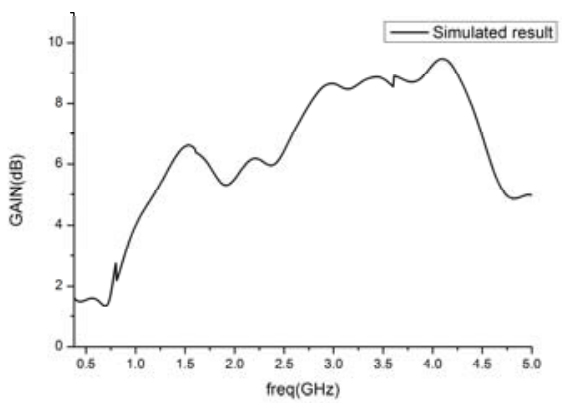

Figure 5. Simulated result of gain varing with frequency 
TABLE I. THE SIMULATED AND MEASURED RESULTS OF THE GAIN

\begin{tabular}{|c|l|c|c|c|c|}
\hline \multirow{2}{*}{ Gain(dB) } & \multicolumn{5}{|c|}{ Frequency(GHz) } \\
\cline { 2 - 6 } & $\mathbf{1 G H z}$ & $\mathbf{2 G H z}$ & $\mathbf{2 . 5 G H z}$ & $3 \mathbf{G H z}$ & $\mathbf{4 G H z}$ \\
\hline Simulated Results & 3.98 & 5.51 & 6.45 & 8.65 & 9.27 \\
\hline Measured Results & 4 & 5.1 & 6.1 & 7.9 & 8.8 \\
\hline
\end{tabular}

The radiation pattern has been tested in the anechoic chamber and the simulated and measured results are also compared in the Fig.6 at the frequency of $1 \mathrm{GHz}, 2.5 \mathrm{GHz}, 3 \mathrm{GHz}$ and $4 \mathrm{GHz}$,respectively.E-plane and $\mathrm{H}-$ plane pattern have been all shown.It can be seen that the simulated results are in good agreement with the measured results. The $3 \mathrm{~dB}$ beamwidth becomes wider in the lowfrequency band than that in the high-frequency band.Better symmetry property between E-plane and H-plane can be obtained as the frequency rises. All patterns show a good radiating directivity.
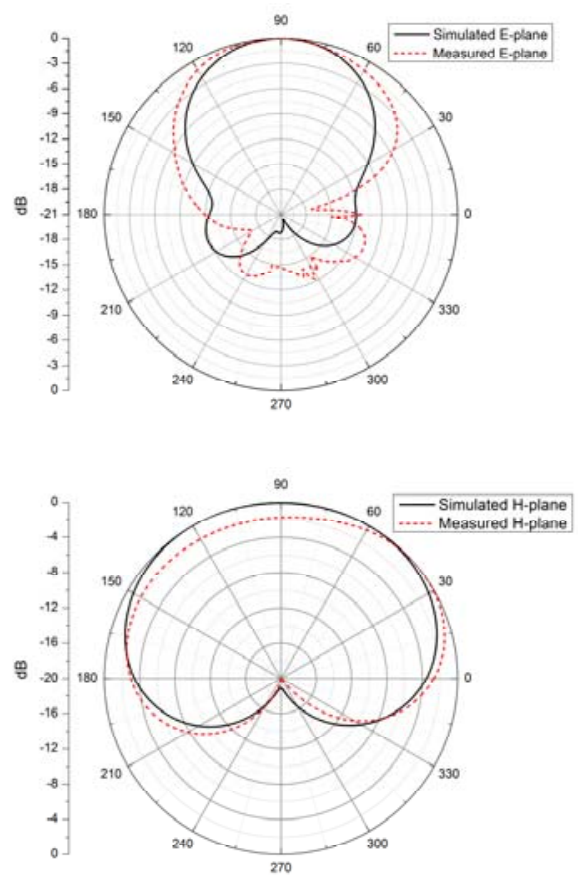

(1)The result at $1 \mathrm{GHz}$

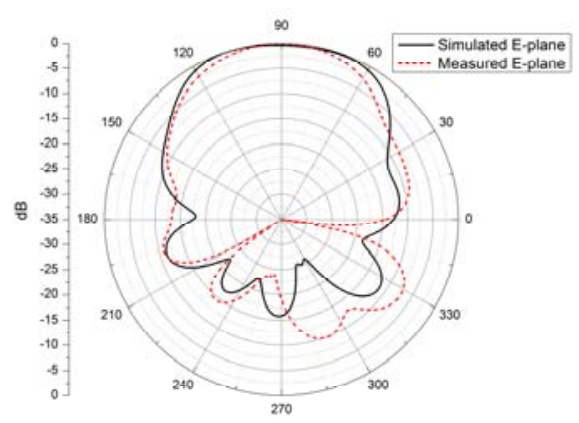

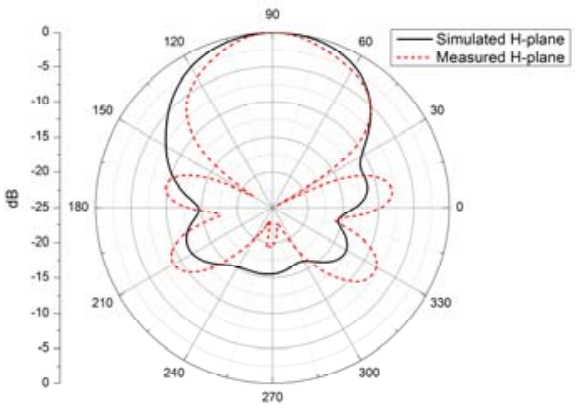

(2)The result at $2.5 \mathrm{GHz}$
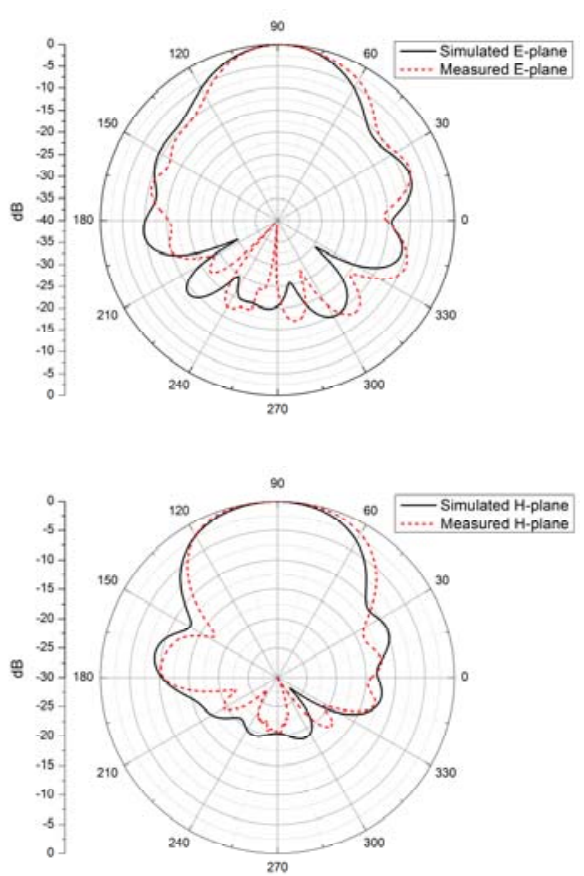

(3)The result at $3 \mathrm{GHz}$

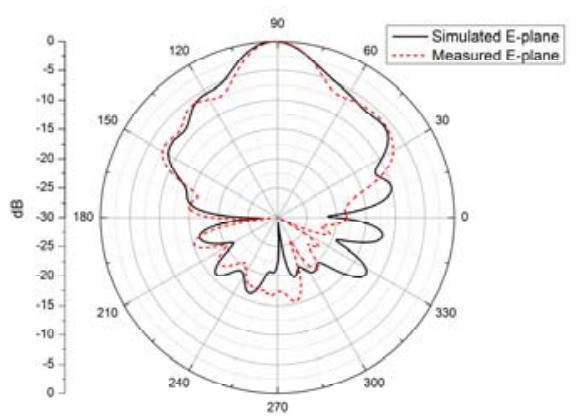




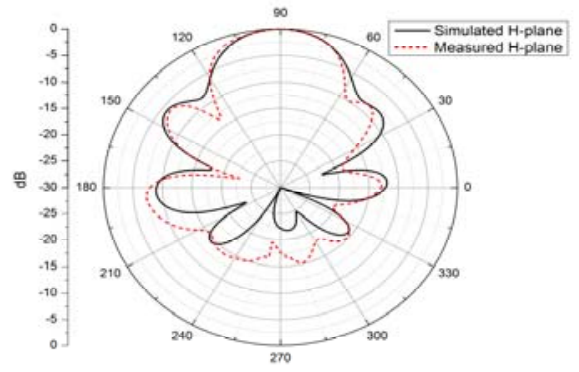

(4)The result at $4 \mathrm{GHz}$

Figure 6. Simulated and measured results of radiation pattern in E-plane and $\mathrm{H}$-plane at the frequency of $1 \mathrm{GHz}, 2.5 \mathrm{GHz}, 3 \mathrm{GHz}$ and $4 \mathrm{GHz}$.

\section{CONCLUSION}

A Vivaldi antenna for ultra-wideband system is designed.By using electromagnetic simulation software HFSS, structure and size of antenna is simulated and optimized. The measured results are agree with the simulated results very well.

The bandwidth for antenna impedance is $0.36-4.8 \mathrm{GHz}$ and size of the antenna has been significantly reduced with loading a resistance, demonstrating that a resistance-loaded method can make the antipodal antenna miniaturization; good radiating directivity is achieved in the entire bandwidth.Because the structure of antenna is simple and parameter fluctuations have less impact on performance,it is easy to process,integrate and form array for this antenna. Therefore, it has wide application prospect in some fields such as ultra-wideband mobile communication antenna, dual-polarized antenna and phased array scanning antenna.

\section{REFERENCES}

[1] P.J.Gibson, "Vivaldi Aerial,"Pro. $9^{\text {th }}$ European Microwave Conf.,Brighton.U.K.,1979,pp.101.

[2] Li Ying, Chen Ai-xin."Design and Application of Vivaldi antenna array," ISAPE 2008.8 $8^{\text {th }}$ International Symposium,2008,pp.261-270.

[3] Gazit.E. "Improved Design of the Vivaldi Antenna."Proc.IEE, 1988,135H,pp.89-9.

[4] Schaubert D H,Chio T H,Holter H. "TSA element design for $500-1500 \mathrm{MHz}$ array. "IEEE Antennas and Propagation Society International Symposium,2000,178-181.

[5] Ehud.Gazit, "Improved design of the Vivaldi antenna," IEEE Proceedings, Vol.135,Pt. H,No.2,April 1988,pp89-92.

[6] Joon Shin,Daniel H.Schaubert. "A Parameter Study of Stripline-Fed Vivaldi Notch-Antenna Arrays," IEEE Transactions on Antenna and Propagation,vol.47, no 5,MAY 1999. 